\title{
Deciding optimal number of exemplars for designing an ECG pattern classifier using MLP
}

R. B. Ghongade ${ }^{1}$ and A. A. Ghatol ${ }^{2}$

\section{${ }^{1}$ Vishwakarma Instt. of Information Technology, Pune, ${ }^{2}$ Dr. Babasaheb Ambedkar Technological Univ, Lonere, India} rbghongade@gmail.com

Abstract: ECG pattern recognition using artificial neural networks is now an established paradigm. Diagnostic systems derive robustness, reliability and speed because of the automatic pattern classifiers. However, a common problem associated with these types of classifiers is to decide the optimal number of exemplars. This paper attempts to find an optimal number of exemplars required for training a multilayer perceptron with acceptable accuracy. Extensive experimentation suggests a figure of 200. Although this figure is specific for multilayer perceptron based classifier, experimentation on similar lines can be performed for other ANN topologies.

Keywords: ECG, MLP, pattern classifier, optimal number of exemplars

Introduction

There are many issues involved in designing an ECG pattern recognition/ classification tool using multilayer perceptrons. One major issue is deciding the number of exemplars for training the artificial neural networks. (Lin He et. al. 2006). This problem is still aggravated due to the large inter-patient variability and the availability of beats. Since ANN based classifiers like MLPs (Gholam Hosseini et. al., 2006) completely depend on the data used for training, it becomes a tricky issue to decide the optimal number of exemplars required for each class. Even the unequal distribution of exemplars in case of multiclass classifiers leads to improper training and generalization is affected. This paper investigates the possibility of determining an optimal number of exemplars per class. A ten-class ECG beat classifier is trained with varying number of exemplars per class and its effect on the test performance is studied.

\section{Material}

Arrhythmia benchmark database from Physionet Massachusetts Institute of Technology -Beth Israel Hospital (MIT-BIH, 2005; MIT-BIH http://ecg.mit.edu) was used for this work. Record numbers 102, 107,109, $111,118,124,200,203,205,207,208,212,213$, $214,217,231,232$ are used for training, cross-validation and testing purposes. A total of $\quad 37,797$ heartbeats were extracted and 29,363 heartbeats were selected.

In this work arrhythmia is the general class of heart disease considered. Arrhythmia presents itself in
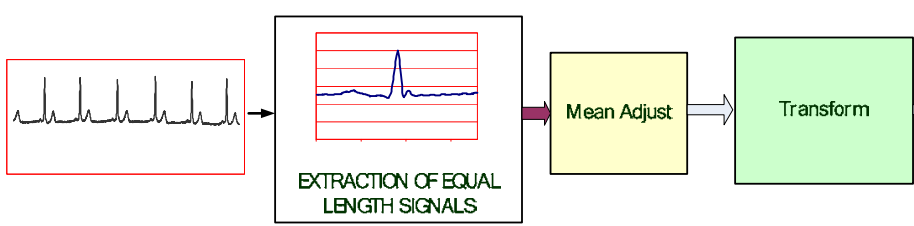
LENGTH SIGNALS

Fig. 1. Block diagram for the classifier the individual heartbeat. The types of arrhythmia to be diagnosed are: Premature Ventricular Contraction (PVC), Left Bundle Branch Block Beat (LBBBB), Right Bundle Branch Block Beat (RBBBB), Atrial Premature Contraction (APC), Fusion of Normal heartbeat and PVC in addition to Normal and Paced beats. Since PVC (also LBBBB and RBBBB) may appear in two different morphological forms, in order to improve the classification accuracy (reduce the complexity of the decision boundaries), we have further divided these in TYPE I and TYPE II categories. Thus there are 10 total types of heartbeats to be classified: APC, FUSION, LBBBB I, NORMAL, PACED, RBBBB I, PVC I, PVC II, LBBBB II and RBBBB II.

\section{Methodology}

Fig.1 depicts the overall methodology for the evaluation and search of the best network. The complete scheme includes various steps like the extraction of equal length ECG signals beat wise, mean adjust to remove the base line wander and dc shift, feature extraction and finally training of the ANN (Jiang \& Kong, 2007)

\section{Data Pre-processing}

Since each record is a continuous waveform it is necessary to extract only a single heartbeat. This is done by considering the $R$ peak and extracting 90 samples on either side of this $R$ peak. It is not mandatory to use all the 180 samples for classification but here we use all 180 samples. Fig. 2 shows the data selection method for one heartbeat. Since the MIT/BIH database comes with annotations for each heartbeat it is necessary to associate the categories with individual extracted beats. A total of 6000 beats were selected with 600 beats belonging to each category viz. APC, FUSION, LBBBB I, NORMAL, PACED, RBBBB I, PVC I, PVC II, LBBBB II and RBBBB II

\section{Feature Extraction}

Feature extraction is an important step so as to reduce the dimensionality of the input space. This

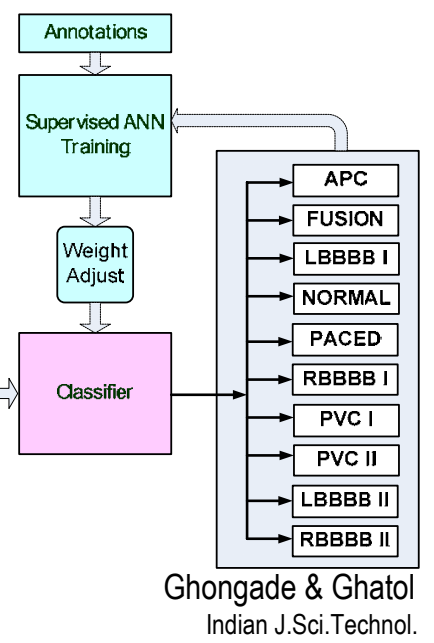


ultimately reduces the required resources (number of neurons) and speeds up the learning. Feature extraction techniques are also numerous (Fabian Vargas et al., 2002; Krishna Prasad \& Shahambi, 2003, Kemal Polat \&

Salih Güneş, 2007) but the real challenge is to have optimum

performance with minimum resources. Principal component analysis, PCA is a traditional approach for feature extraction. Other statistical features like R-peak, Mean power spectral density and area under QRS have also been explored (Fabian Vargas et al., 2002). Here we propose using discrete cosine transform for feature extraction. The mean adjusted data obtained in previous process is then subjected to discrete cosine transformation. A 180 coefficient data is obtained because of this. Here we can use the energy compaction property of the DCT to reduce

\begin{tabular}{|c|c|}
\hline \multicolumn{2}{|c|}{$\begin{array}{c}\text { Table1. DCT coefficients and the } \\
\text { percent root mean difference }\end{array}$} \\
\hline DCT Coefficients & PRD \% \\
\hline 5 & 3.9627 \\
\hline 15 & 1.3229 \\
\hline 30 & 0.58804 \\
\hline
\end{tabular}

Fig. 2. Extraction of individual heart beat using R peak

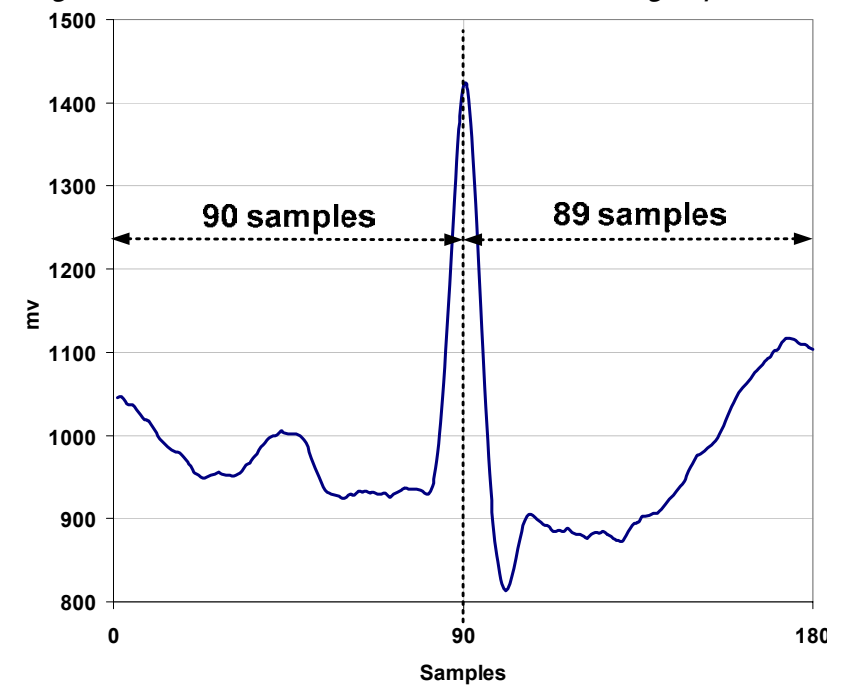

Neural Network Models Multilayer perceptrons (MLPs) are layered feedforward networks typically Reconstruction Errors for DCT

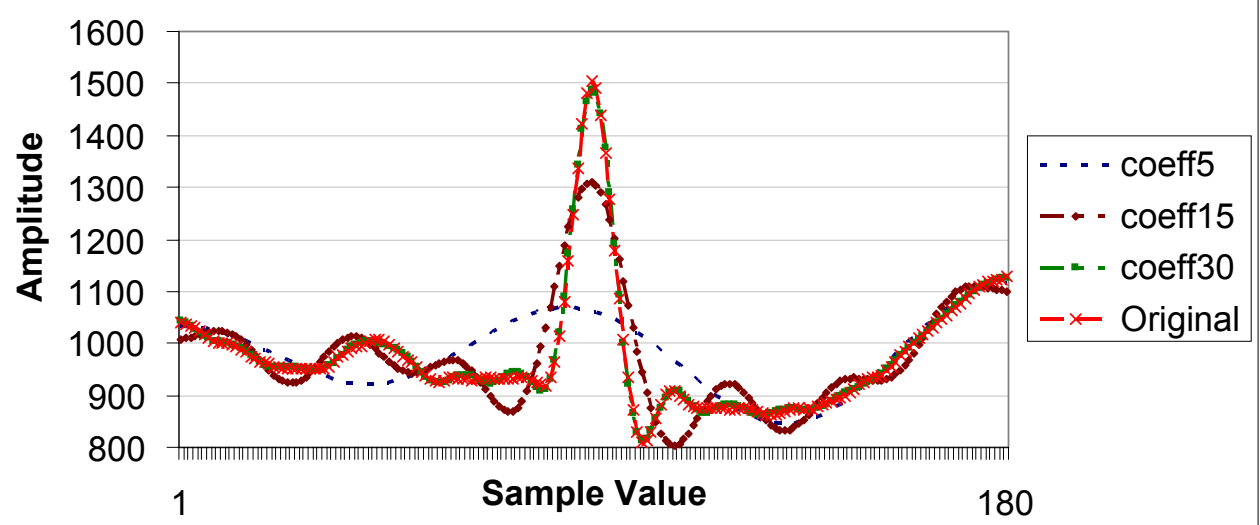

Fig. 3. Reconstruction errors due to DCT coefficients

trained with static backpropagation. These networks have found their way into countless applications requiring static pattern classification. Their main advantage is that they are easy to use, and that they can approximate any input/output map. The key disadvantages are that they train slowly, and require lots of training data (typically three times more training samples than network weights). This work uses one hidden layer MLP with momentum learning and tan-sigmoidal activation functions for hidden as well as

the dimensions of the data. Only those dominant components are retained, which contribute most to the signal energy. First 30 coefficients were found to contribute to about $99.49 \%$ of the total energy. In addition to this the percent root mean difference (PRD) was calculated by reconstructing the normal waveform from only 30 components and comparing it with the original normal waveform (Fabian Vargas et al., 2002, Tadejko \& Rakowski Waldemar, 2007). Table 1 depicts the relationship between number of DCT coefficients selected and PRD. Fig. 3 depicts the reconstruction errors due to truncation of DCT coefficients. This PRD metric is important since we want to preserve the morphological features of the heart beat (Fabian Vargas et al., 2002, Rahime Ceylan \& Yüksel Özbay, 2007). It was observed that a minimum $30 \mathrm{DCT}$ coefficients were required to preserve the morphological information. output layers. Fig. 4 depicts the MLP structure.

Experimentation and Results

The feature vector of 30 DCT coefficients per heartbeat is used as input data and the corresponding annotation forms the target. Thus the dataset consists of 6000 input-target pairs.

\section{Experiment 1}

A multilayer perceptron is employed with backpropagation and momentum learning. The first experiment was to find optimum number of hidden layer neurons thus deciding the network configuration. For this the number of exemplars for each class was kept equal and the dataset was divided into training, cross-validation and testing sets with a ratio of $50 \%, 25 \%$ and $25 \%$ respectively. The number of hidden layer neurons was varied from 15 through 100. Each training-testing session was carried out for five times thus effecting multistart
Research article

CIndian Society for Education and Environment (iSee)
"Designing an ECG"

http://www.indjst.org
Ghongade \& Ghatol Indian J.Sci.Technol. 


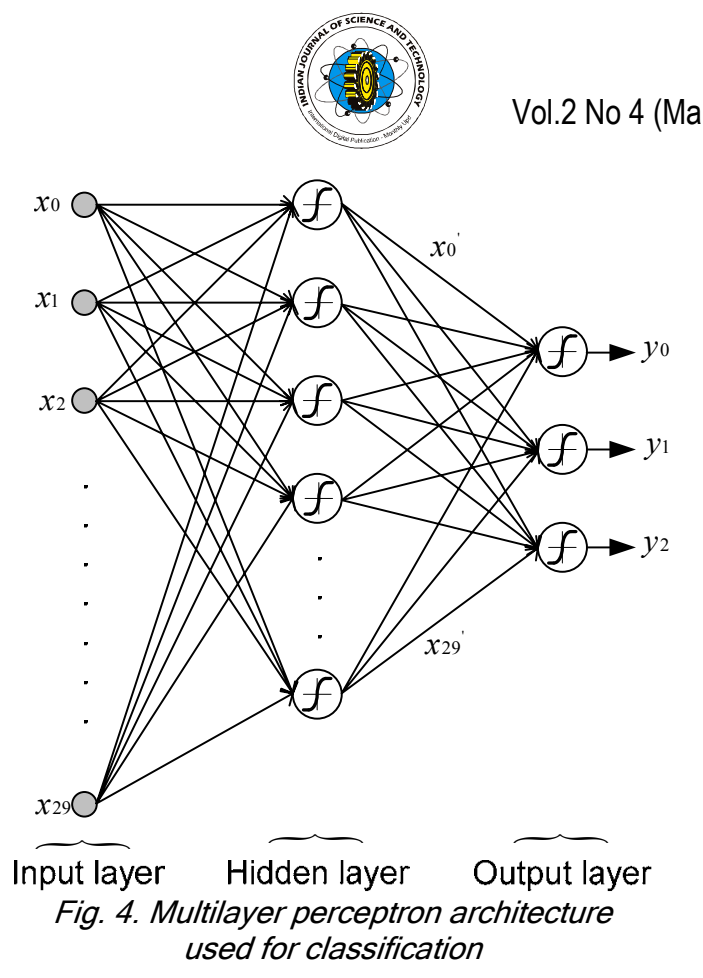

training. The best network was thus found as MLP with 80 hidden layer neurons which exhibited an average accuracy of $99.1 \%$. This network i.e; $30 \times 80 \times 10$ is used for the second experiment Experiment 2

In this experiment the dataset is modified so as to have unequal number of exemplars for training. Separate datasets with biased probabilities were thus created corresponding to 10 classes where the exemplar numbers varied from 1 through 300 in variable steps for each class at a time. The network was again trained and tested for five times and the average accuracy was calculated for the biased class. Fig. 5 depicts the results of the experiment.

Conclusion and discussion

\section{Training Exemplars v/s Classisfication Accuracy}

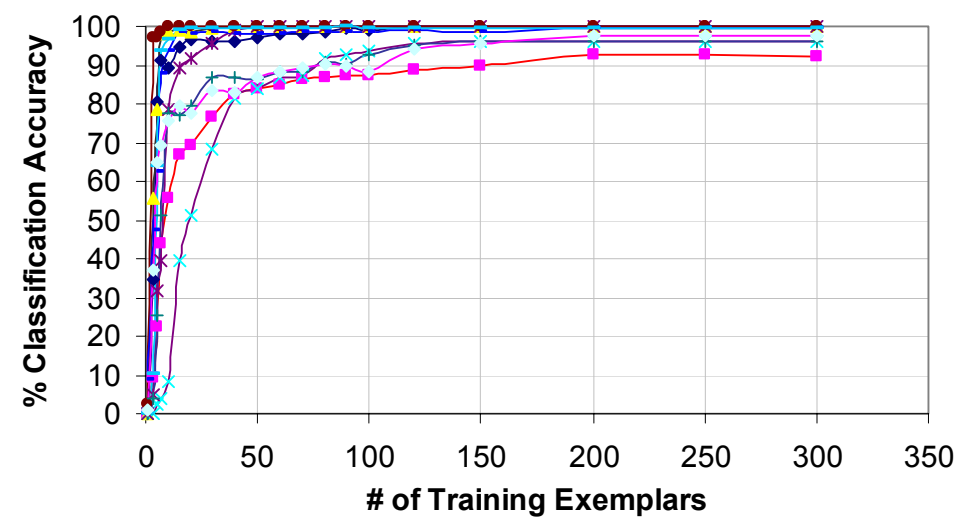

Fig.5. Number of training exemplars and the corresponding classifier accuracy

It can be seen from Fig. 5 that the classifier accuracy depends on the number of exemplars used for training. As more number of exemplars is available for training the MLP, the classification accuracy increases but ultimately a saturation value is reached, after which an increase in the number of exemplars does not significantly affect the classifier accuracy. This point where the accuracy saturates can be considered as the threshold number for determining the optimal number of exemplars required for training. In the case of a ten class ECG beat classifier employing MLP, this number is 200 .

\section{References}

1. Fabian Vargas, Djones Lettin, Maria Cristina Felippetto de Castro, Marcello Macarthy (2002) Electrocardiogram pattern recognition by means of MLP network and PCA: a case study on equal

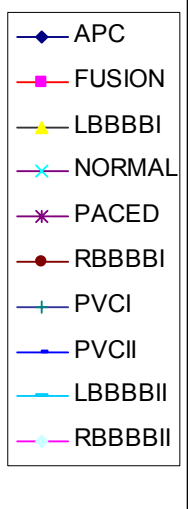
and least square support vector machine. Appl. Maths. \& Comput. 186 (1), 898-906.

5. Krishna Prasad G, Shahambi JS (2003) Classification of ECG arrhythmias using multi-resolution analysis and neural networks, IEEE, 1, 227-231.

6. Lin He, Wensheng Hou, Xiaolin Zhen and Chenglin Peng (2006) Recognition of ECG patterns using artificial neural network. 6th Intl. Conf. on Intelligent Systems Design \& Applns. 2, 477- 481.

7. Rahime Ceylan and Yüksel Özbay (2007) Comparison of FCM, PCA and WT techniques for classification ECG arrhythmias using artificial neural network. Expert Systems with Applns. 33, 286-295.

8. Tadejko Pawel and Rakowski Waldemar (2007) Mathematical morphology based ECG feature extraction for the purpose of heartbeat classification. 6th Intl. Conf. on Computer Info. Systems \& Industrial Mangt. Applns. pp: 322-327.
Ghongade \& Ghatol Indian J.Sci.Technol. 